\title{
Author Correction: Gate controlling of quantum interference and direct observation of anti-resonances in single molecule charge transport
}

Yueqi Li, Marius Buerkle, Guangfeng Li, Ali Rostamian, Hui Wang, Zixiao Wang, David R. Bowler, Tsuyoshi Miyazaki, Limin Xiang, Yoshihiro Asai, Gang Zhou and Nongjian Tao

Correction to: Nature Materials https://doi.org/10.1038/s41563-018-0280-5, published online 11 February 2019.

In the version of this Article originally published, the subthreshold swing was incorrectly given throughout as $17 \mathrm{mV}^{\mathrm{dec}}{ }^{-1}$; it should have been $170 \mathrm{mV} \mathrm{dec}^{-1}$. Statements about the subthreshold values have been removed and the following text changes have been made.

At the end of the abstract, the text 'we achieve continuous control of the conductance over two orders of magnitude with a subthreshold

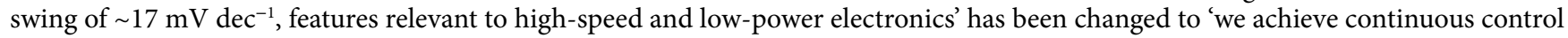
of the conductance over two orders of magnitude, demonstrating a different gating mechanism to conventional field-effect transistors'.

In the third from last paragraph of the main text, the text 'the subthreshold swing is limited by fundamental thermodynamics and is greater than $\sim 60 \mathrm{mV}$ at room temperature. In the present case, the subthreshold swing is determined by quantum interference, rather than thermodynamics, and the value estimated from the conductance change between $-0.55 \mathrm{~V}$ and $-0.45 \mathrm{~V}$ is $17 \mathrm{mV}$ dec ${ }^{-1}$, which is substantially smaller than in conventional devices' has been changed to 'gating originates from the control of carrier density in the conduction channel and its efficiency is subject to the laws of thermodynamics. In the present case, the gating principle is quantum interference, which is different to the conventional semiconductor devices'.

In the final paragraph of the main text, the text 'which have a minimum subthreshold swing of $\sim 60 \mathrm{mV} \mathrm{dec}{ }^{-1}$, a limit originating in ther-

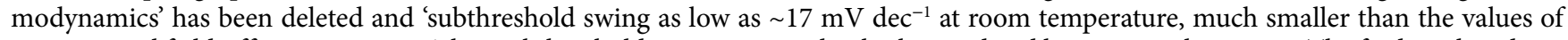
conventional field-effect transistors. A low subthreshold swing is critical to high-speed and low-power electronics. The finding thus demonstrates a unique feature of quantum interference' has been changed to 'large change in single-molecule conductance, which suggests a different gating principle to the conventional field-effect transistors'.

These changes have been made to the online versions of the Article and do not affect its conclusions.

Published online: 13 November 2019

https://doi.org/10.1038/s41563-019-0552-8

(c) The Author(s), under exclusive licence to Springer Nature Limited 2019 